\title{
Microscopic theory for the light-induced anomalous Hall effect in graphene
}

\author{
S. A. Sato, ${ }^{1,2, *}$ J. W. McIver, ${ }^{2}$ M. Nuske, ${ }^{3}$ P. Tang, ${ }^{2}$ G. Jotzu, ${ }^{2}$ B. Schulte, ${ }^{2}$ H. Hübener, ${ }^{2}$ \\ U. De Giovannini, ${ }^{2}$ L. Mathey, ${ }^{3,4}$ M. A. Sentef, ${ }^{2}$ A. Cavalleri, ${ }^{2}$ and A. Rubio ${ }^{2,5, \dagger}$ \\ ${ }^{1}$ Center for Computational Sciences, University of Tsukuba, 1-1-1 Tennodai, Tsukuba, Ibaraki, Japan \\ ${ }^{2}$ Max Planck Institute for the Structure and Dynamics of Matter, Luruper Chaussee 149, 22761 Hamburg, Germany \\ ${ }^{3}$ Zentrum für Optische Quantentechnologien and Institut für Laserphysik, Universität Hamburg, Luruper Chaussee 149, \\ 22761 Hamburg, Germany \\ ${ }^{4}$ The Hamburg Centre for Ultrafast Imaging, University of Hamburg, Luruper Chaussee 149, 22761 Hamburg, Germany \\ ${ }^{5}$ Center for Computational Quantum Physics (CCQ), Flatiron Institute, 162 Fifth Avenue, New York, New York 10010, USA
}

(Received 20 January 2019; published 10 June 2019)

\begin{abstract}
We employ a quantum Liouville equation with relaxation to model the recently observed anomalous Hall effect in graphene irradiated by an ultrafast pulse of circularly polarized light. In the weak-field regime, we demonstrate that the Hall effect originates from an asymmetric population of photocarriers in the Dirac bands. By contrast, in the strong-field regime, the system is driven into a nonequilibrium steady state that is well described by topologically nontrivial Floquet-Bloch bands. Here, the anomalous Hall current originates from the combination of a population imbalance in these dressed bands together with a smaller anomalous velocity contribution arising from their Berry curvature. This robust and general finding enables the simulation of electrical transport from light-induced Floquet-Bloch bands in an experimentally relevant parameter regime and creates a pathway to designing ultrafast quantum devices with Floquet-engineered transport properties.
\end{abstract}

DOI: 10.1103/PhysRevB.99.214302

Optical control of functional materials has emerged as an important research front bridging condensed matter physics [1] and ultrafast spectroscopy [2]. Many noteworthy phenomena have been discovered in optically driven quantum solids, including light-induced superconductivity [3,4], various types of photo-initiated insulator-metal transitions [5-8], light control of microscopic interactions like electronphonon coupling [9-11], and theoretically predicted Floquettopological phases of matter [12-16]. Floquet-topological phases in particular have stimulated much interest but direct evidence of electron-photon Floquet-dressed states in solids is scarce to date $[17,18]$, in contrast to the field of artificial lattices [19-35].

Recently, a light-induced anomalous Hall effect was observed in graphene using ultrafast transport techniques [36]. A key challenge for the interpretation of the reported effects lies in the competition between Floquet engineering of Hamiltonians versus the role of electronic population effects. For the case of laser-driven graphene, the latter are particularly important as the pump laser is generically resonant with electronic excitations. Here we provide a theoretical framework within which this class of experiments [36] can be interpreted.

A graphene lattice subjected to circular driving has been studied theoretically in a variety of frameworks [12-15,22,37-43]. We focus here on the low-frequency driving regime and find that the driven-dissipative dynamics together with the applied bias field plays a crucial role

\footnotetext{
*ssato@ccs.tsukuba.ac.jp

†angel.rubio@mpsd.mpg.de
}

in understanding the transport properties of the Floquetengineered state. Our real-time simulations contain both the population imbalance of excited photocarriers in the Dirac cone of graphene as well as the Floquet-topological Berry curvature of photon-dressed bands. We find that population effects play an important role under the low-frequency driving used in the experiments in both weak and strong driving limits. In the weak-driving regime, light-induced Hall transport originates mainly from population imbalance of photocarriers in the bare bands. By contrast, in the strong field regime clear topological-Floquet states are formed, and the light-induced Hall effect is characterized by a population imbalance of these Floquet bands that outweighs, however, the Floquet-Berry curvature contribution which is predicted to dominate in the high-frequency regime $[12,38,41]$. Our results demonstrate that Floquet engineering in solids is a reality, even with significant dissipation. These findings are in good agreement with the experimental results [36] and provide a microscopic interpretation of the observed light-induced anomalous Hall effect.

To model the electron dynamics in graphene under electromagnetic fields, we employ a quantum Liouville equation for the one-particle reduced density matrix $\rho_{\boldsymbol{K}(t)}$,

$$
\frac{d}{d t} \rho_{\boldsymbol{K}(t)}(t)=\frac{\left[H_{\boldsymbol{K}(t)}, \rho_{\boldsymbol{K}(t)}(t)\right]}{i \hbar}+\hat{D}_{\boldsymbol{K}(t)} \rho_{\boldsymbol{K}(t)}(t),
$$

with phenomenological relaxation $\hat{D}_{\boldsymbol{K}(t)}$ and the Dirac Hamiltonian, $H_{\boldsymbol{K}(t)}=\hbar v_{F} \tau_{z} \sigma_{x} K_{x}(t)+\hbar v_{F} \sigma_{y} K_{y}(t)$, where $v_{F}$ is the Fermi velocity, $\sigma_{x / y}$ are the Pauli matrices, and $\boldsymbol{K}(t)=\boldsymbol{k}+$ $\boldsymbol{e A}(t) / \hbar c$ is the lattice momentum coupled to an external vector potential $\boldsymbol{A}(t)$. The linear Dirac-cone approximation is 
justified in the present context of low-frequency driving and moderate deviations from half-filled bands with a chemical potential shift $\mu$ [36]. The different chirality of the Dirac fermions at the $K$ and $K^{\prime}$ points is given by $\tau_{z}= \pm 1$. The phenomenological dissipation $\hat{D}_{\boldsymbol{K}(t)}$ based on the relaxation time approximation is added to account for relaxation and dephasing effects [44] (see also Appendix A). We note that effects of dissipatively coupled Floquet-topological systems have been discussed previously [40-42,45], but not in the concrete context of the present paper. Here we set the relaxation time $T_{1}$ to $100 \mathrm{fs}$, and the dephasing time $T_{2}$ to $20 \mathrm{fs}$. However, the qualitative behavior of the light-induced Hall effect does not strongly depend on the choice of $T_{1}$ and $T_{2}$.

To evaluate the Hall conductivity $\sigma_{x y}$, we apply a weak static source-drain electric field along the $y$ axis of the model and compute the transverse current along the $x$ axis under the presence of a circular laser pulse. Both the static electric field and the laser pulse are included in the model via the vector potential $\boldsymbol{A}(t)$. Following the same analysis as in Ref. [36], we define the Hall current as the difference of the transverse current induced by right- and left-handed circular laser fields (pump dichroism, see also Appendix B). To match experimental values, we set the wavelength of the circular laser pulse to $\lambda=6.5 \mu \mathrm{m}$, which corresponds to the mean photon energy of $\hbar \omega_{\text {MIR }} \approx 190 \mathrm{meV}$. The pulse duration is $1 \mathrm{ps}$ (FWHM). Note that the present model leads to the identical Hall current around $K$ and $K^{\prime}$ with $\tau_{z}= \pm 1$.

First, we investigate the light-induced Hall effect in the weak-driving regime, $E_{\mathrm{MIR}}=1 \mathrm{MV} / \mathrm{m}$. Figure 1 (a) shows the computed Hall conductivity $\sigma_{x y}$ as a function of $\mu$, while Fig. 1(b) shows the experimental Hall conductivity with the weakest experimental fluence $0.01 \mathrm{~mJ}$ [36]. Our results confirm that the Hall conductivity is proportional to the laser intensity in this regime. Therefore, the single-photon absorption process is expected to play a dominant role in the light-induced Hall effect. Indeed, both the theoretical and experimental results consistently show a strong suppression of the Hall effect once $\mu$ reaches $\pm \hbar \omega_{\mathrm{MIR}} / 2$, which is when single-photon absorption becomes suppressed. The experimental result shows a sign change of the Hall conductivity in the higher doping regime while the theoretical result shows the same sign in the whole chemical potential range. The signchange feature might be understood by a negative offset due to the inverse Faraday effect from a substrate [46]. However, since a detailed analysis of the substrate effect is beyond the scope of the present paper, this aspect will be investigated in the future.

To clarify the microscopic origin of the Hall current in the weak field regime, we perform a perturbative analysis which shows that the Hall current originates from population imbalance of photocarriers in the Brillouin zone (BZ) (see Appendix E). An excess of photocarriers is generated on one side of the Dirac cone compared to the other, and the nonsymmetric photocarrier-distribution results in a net Hall current. Furthermore, the perturbative analysis reveals that the population imbalance is induced by the interference of two excitation paths: one of them is the single-photon absorption process in the bare Dirac band [black-dashed line in Fig. 1(c)], while the other one is the single-photon absorption in the tilted Dirac band [blue solid line in Fig. 1(c)],
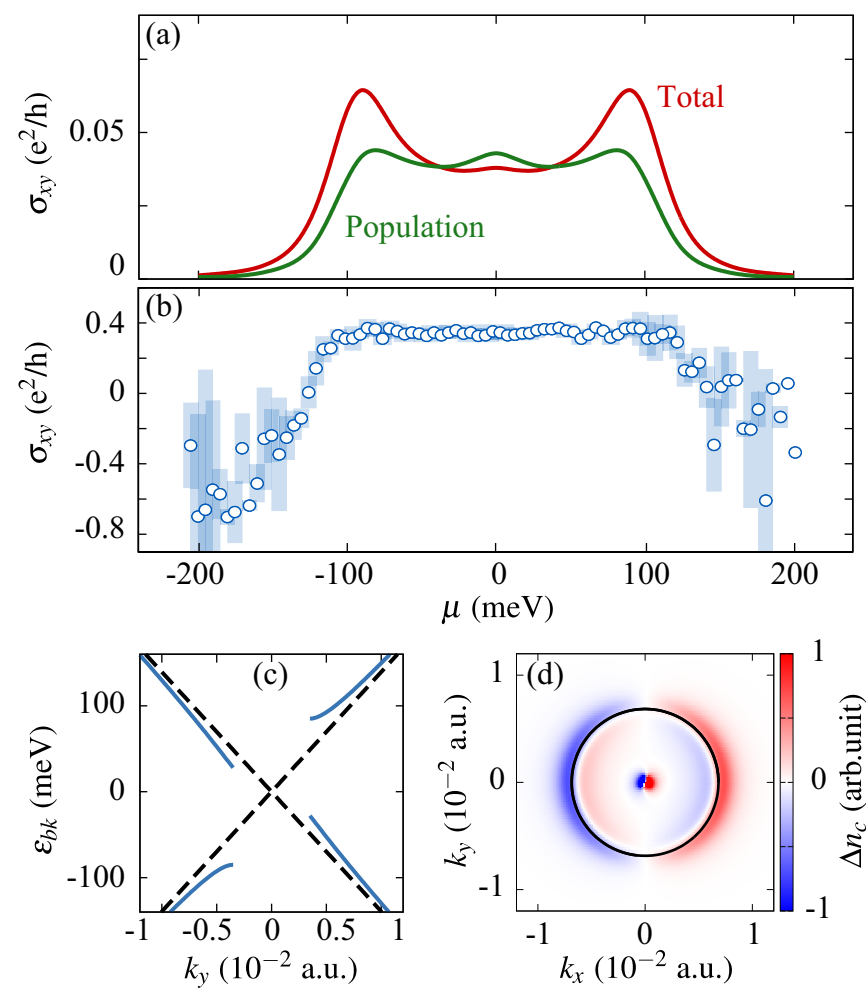

FIG. 1. Light-induced Hall conductivity in the weak-field regime, $E_{\mathrm{MIR}}=1 \mathrm{MV} / \mathrm{m}$ : (a) Theoretical Hall conductivity $\sigma_{x y}$ as a function of $\mu$. The full simulation result (red) and the population contribution (green) are shown. (b) The experimental Hall conductivity with the peak laser fluence of $0.01 \mathrm{~mJ} / \mathrm{cm}^{2}$ as shown in Ref. [36]. (c) Electronic structure of the Dirac Hamiltonian. Blackdashed lines show the original Dirac bands, while blue-solid lines show the tilted Dirac bands under a source-drain field with strength 0.2 a.u. (d) Pump dichroism of conduction-band populations under source-drain bias along the $y$ direction.

where the tilt is induced by the static source drain field. To confirm this conclusion, we further compute the conduction band population $\rho_{c c, \boldsymbol{K}(t)}(t)=\operatorname{Tr}\left[\left|u_{c \boldsymbol{K}(t)}^{s}\right\rangle\left\langle u_{c \boldsymbol{K}(t)}^{s}\right| \rho_{\boldsymbol{K}(t)}(t)\right]$ using instantaneous eigenstates of the Hamiltonian; $\hat{H}_{K(t)}\left|u_{b K(t)}^{s}\right\rangle=$ $\epsilon_{b \boldsymbol{K}(t)}\left|u_{b \boldsymbol{K}(t)}^{s}\right\rangle$, where $b$ denotes the band index, $v$ and $c$ for valence and conduction bands, respectively. Figure 1(d) shows the cycle-averaged conduction-band population pump dichroism. A population imbalance along the $x$ direction is clearly observed under the source-drain field along the $y$ direction. From this, we compute the population contribution to the Hall current, $J_{H}^{\mathrm{POP}}(t)$ by multiplying populations with corresponding band velocities:

$$
J_{H}^{\mathrm{POP}}(t)=-\frac{2 e}{\hbar(2 \pi)^{2}} \sum_{b=v, c} \int d \boldsymbol{k} \frac{\partial \epsilon_{b \boldsymbol{K}(t)}}{\hbar \partial k_{x}} \rho_{b b, \boldsymbol{K}(t)} .
$$

The result shown in Fig. 1(a) accounts for most of the total Hall conductivity. Overall, our analysis demonstrates that in the weak-field regime the Hall current mainly originates from a population imbalance of photocarriers in the bare Dirac band. Importantly, the population imbalance mechanism does not rely on the specific properties of the Dirac Hamiltonian. Therefore, the mechanism is general and can induce 

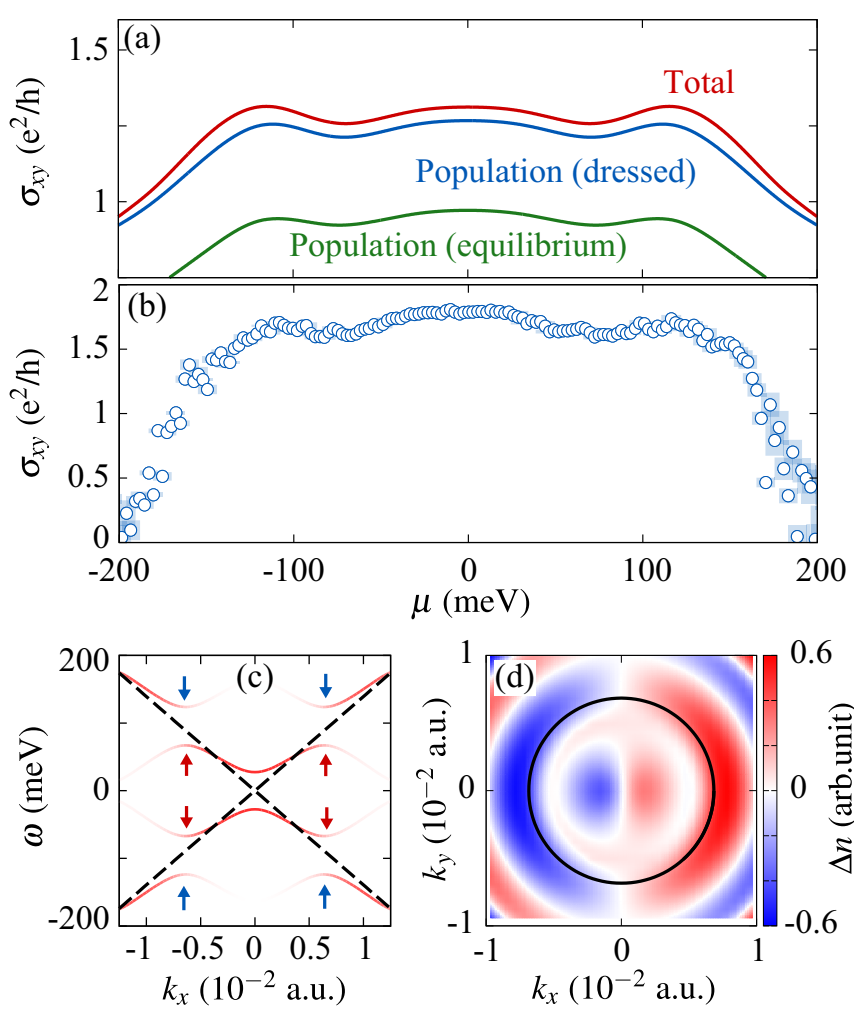

FIG. 2. Light-induced Hall conductivity in the strong field regime, $E_{\mathrm{MIR}}=20 \mathrm{MV} / \mathrm{m}$ : (a) The theoretical Hall conductivity $\sigma_{x y}$ as a function of $\mu$. The full simulation result (red), the population contribution in the original Dirac band (green), and the naturalorbital population contribution (blue) are shown. (b) The experimental Hall conductivity with the peak laser fluence of $0.23 \mathrm{~mJ} / \mathrm{cm}^{2}$ as shown in Ref. [36]. (c) Floquet bands (red) and the original Dirac cone (black dashed). Outer (inner) edges of the resonant gap are indicated by blue (red) arrows. (d) Pump dichroism of natural-orbital population with source-drain bias along $y$ direction.

anomalous Hall currents even in conventional semiconductors (see Appendix F).

Having established an asymmetric population imbalance in the Dirac bands as the source of light-induced anomalous Hall currents in the weak-field regime, we move on to explore the strong optical driving regime. We set $E_{\mathrm{MIR}}$ to $20 \mathrm{MV} / \mathrm{m}$, which is the peak field intensity for the largest reported experimental fluence, $0.23 \mathrm{~mJ}$ [36]. Figure 2(a) shows the computed Hall conductivity $\sigma_{x y}$ as a function of $\mu$, while Fig. 2(b) shows the corresponding experimental result [36]. The theoretical and experimental data are in good agreement, with both exhibiting conductivity maxima around $\mu=0$ as well as $\pm \hbar \omega_{\mathrm{MIR}} / 2$. Figure 2(c) shows the calculated Floquet band structure for the same driving parameters. The Floquet bands show characteristic gap openings at $\epsilon_{b \boldsymbol{k}}=0$, and $\pm \hbar \omega_{\mathrm{MIR}} / 2$. Strikingly, the peaks in $\sigma_{x y}$ near $\pm \hbar \omega_{\mathrm{MIR}} / 2$ coincide with the outer edges of the Floquet bands [blue arrows in Fig. 2(c)], and the width of the central plateau of $\sigma_{x y}$ is approximately the width of the gap at the Dirac point. This already indicates a close relation between the formation of Floquet-Bloch bands and the anomalous Hall effect for strong laser driving.

To clarify the role of Floquet-Bloch states in the generation of anomalous Hall currents, we analyze to what extent they represent the full dynamics of the system under the driving conditions. Since the complete dynamics is described by the time-dependent density matrix $\rho_{\boldsymbol{K}(t)}$, we analyze the time evolution in terms of its instantaneous eigenstates, the time-dependent natural orbitals $\left|u_{b k}^{\mathrm{NO}}(t)\right\rangle$ [47], giving $\rho_{\boldsymbol{K}(t)}=$ $\sum_{b=v, c} n_{b \boldsymbol{k}}^{\mathrm{NO}}(t)\left|u_{b \boldsymbol{k}}^{\mathrm{NO}}(t)\right\rangle\left\langle u_{b \boldsymbol{k}}^{\mathrm{NO}}(t)\right|$. This orbital basis for the dynamics gives the full dressed states of the system without the assumptions required by Floquet theory and allows us to make a detailed assessment of the Floquet picture.

The cycle-averaged population of the natural orbitals is computed as $\tilde{n}_{b k}^{\mathrm{NO}}=\int_{0}^{T_{\mathrm{MIR}}} d t n_{b \boldsymbol{k}}^{\mathrm{NO}}(t) / T_{\mathrm{MIR}}$ and Fig. 2(d) shows the pump dichroism of the natural-orbital population $\tilde{n}_{b k}^{\mathrm{NO}}$. Consistent with the population imbalance in the weak-field regime [Fig. 1(d)], the natural-orbital population also shows the imbalance along the $x$ direction. From the population of the natural orbitals, we can compute the dressed-band population contribution to the Hall current as

$$
J_{H}^{D-\mathrm{POP}}=-\frac{2 e}{\hbar(2 \pi)^{2}} \sum_{b=v, c} \int d \boldsymbol{k} \tilde{v}_{b \boldsymbol{k}}^{\mathrm{NO}} \tilde{n}_{b \boldsymbol{k}}^{\mathrm{NO}},
$$

where $\tilde{v}_{b k}^{\mathrm{NO}}$ is an effective dressed-band natural-orbital velocity, $\tilde{v}_{b \boldsymbol{k}}^{\mathrm{NO}}=v_{F} \int_{0}^{T_{\mathrm{MIR}}} d t\left\langle u_{b \boldsymbol{k}}^{\mathrm{NO}}(t)\left|\sigma_{y}\right| u_{b \boldsymbol{k}}^{\mathrm{NO}}(t)\right\rangle / T_{\mathrm{MIR}}$. The blue curve in Fig. 2(a) shows the contribution of the natural-orbital population computed by Eq. (3). The contribution of the natural-orbital population reproduces the full signal very well in the whole investigated chemical-potential range, indicating that a dressed state picture gives a valid description of the light-induced Hall current. As a reference, we also evaluated the contribution from the population imbalance in the bare Dirac band with Eq. (2) and show the result as the green curve in Fig. 2(a). From the results, it is clear that the population imbalance in the bare Dirac band is not sufficient to describe the full signal. Thus we confirmed that the dressed states describe a significantly different electronic structure and that the light dressing enhances the light-induced Hall effect.

Having established the role of dressed bands in the strong-field regime, we turn to the relation between Floquet states and natural orbitals under continuous circular laser fields without the source-drain field. For this purpose, we introduce the Floquet fidelity, $S_{k}$, as a measure of similarity between Floquet states $\left|u_{b k}^{F}(t)\right\rangle$ and natural orbitals $\left|u_{b k}^{\mathrm{NO}}(t)\right\rangle$. The Floquet fidelity is defined as the absolute value of the determinant of the fidelity matrix, $S_{k}=\left|\operatorname{det} F_{k}\right|$, where each element of the matrix $F_{k}$ is the cycle-averaged overlap between natural orbitals and Floquet states, $F_{\boldsymbol{k}, i j}=$ $\int_{0}^{T_{\mathrm{MIR}}} d t\left|\left\langle u_{i k}^{\mathrm{NO}}(t) \mid u_{j k}^{F}(t)\right\rangle\right|^{2} / T_{\mathrm{MIR}}$ (see also Appendix $\mathrm{H}$ ). The Floquet fidelity $S_{k}$ can take a maximum value of one only if the natural orbitals and the Floquet states are identical.

Figure 3(a) shows the Floquet fidelity $S_{k}$ at the Dirac point as a function of the driving field strength. The system is far from the Floquet limit in the weak-field regime $\left(S_{k} \approx 0\right)$, while it approaches the Floquet limit in the strong-field regime $\left(S_{k} \approx 1\right)$. This behavior is consistent with the above findings: The population imbalance in the bare Dirac band dominates the Hall current in the weak-field regime, while the dressedstate picture is more appropriate in the strong-field regime.

A map of the Floquet fidelity $S_{k}$ in the BZ in the strong field regime is shown in the inset of Fig. 3(a), where the single-photon resonance, $\hbar v_{F}|\boldsymbol{k}|=\hbar \omega_{\mathrm{MIR}} / 2$, is indicated as a 

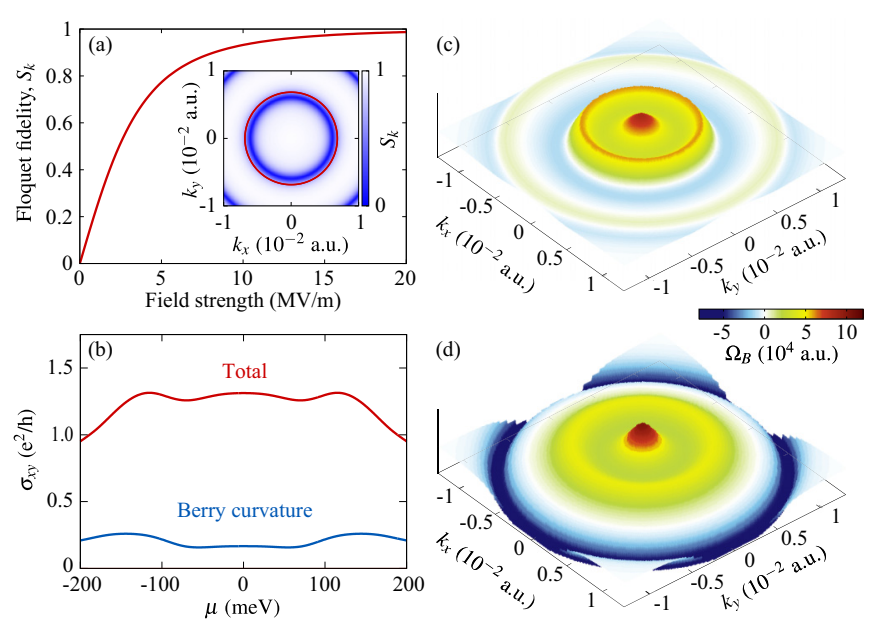

FIG. 3. Relation of the steady state orbitals and the Floquet states: (a) Floquet fidelity, $S_{k}$, at the Dirac point, $\boldsymbol{k}=0$, as a function of the driving field strength. The inset shows the Floquet fidelity in the $\mathrm{BZ}$ in the strong field regime where $E_{\mathrm{MIR}}=20 \mathrm{MV} / \mathrm{m}$. (b) Comparison between the full conductivity $\sigma_{x y}$ and the Berry curvature contribution $\sigma_{x y}^{T}$. (c) The Berry curvature of the steady-state natural orbitals in the strong field regime. (d) The Berry curvature of the corresponding Floquet states.

red circle. This result shows that Floquet states are established throughout a large portion of the BZ including the Dirac point, except for a ring close to the the single-photon resonance $\left(S_{k} \approx 1\right)$, where the steady state appears to be strongly disturbed $\left(S_{k} \approx 0\right)$. In relation to the Floquet-bandstructure in Fig. 2(c), this indicates that the circular laser field is able to establish the outer edges of the Floquet states [the blue arrows in Fig. 2(c)], while the inner edges (red arrows) do not form. Moreover, the realization of the outer Floquet edges is supported by the appearance of the peaks in Fig. 2(a) that are positioned at the resonance energies.

Having demonstrated the relevance of a Floquet dressed-state picture under strong-field driving, we now discuss the role of Berry curvature of the dressed states for the Hall current. We compute the cycle-averaged Berry curvature of the natural orbitals, $\Omega_{B}^{\mathrm{NO}}(\boldsymbol{k})=$ $-i \int_{0}^{T_{\mathrm{MIR}}} d t\left[\nabla_{\boldsymbol{k}} \times\left\langle u_{b k}^{\mathrm{NO}}(t)\left|\nabla_{\boldsymbol{k}}\right| u_{b k}^{\mathrm{NO}}(t)\right\rangle\right]_{z} / T_{\mathrm{MIR}} \quad$ shown in Fig. 3(c), while Fig. 3(d) shows the Berry curvature of the corresponding Floquet state at $E_{\mathrm{MIR}}=20 \mathrm{MV} / \mathrm{m}$. Both the natural orbitals and the Floquet state consistently show a positive Berry curvature at the Dirac point $(\boldsymbol{k}=0)$ and at the first resonance $\left(v_{F}|\boldsymbol{k}|=\hbar \omega_{\mathrm{MIR}} / 2\right)$. Indeed, the natural-orbital Berry curvature integrated over the BZ is found to be $\int d \boldsymbol{k} \Omega_{B}^{\mathrm{NO}}(\boldsymbol{k})= \pm \pi$ for each Dirac cone, which is consistent with the topological Floquet-Chern insulator picture [12].

Finally, we quantify the Hall currents expected solely from the time-averaged Berry curvature of the bands by computing the Hall conductivity [12]:

$$
\sigma_{x y}^{T}=\frac{2 e^{2}}{\hbar} \int \frac{d \boldsymbol{k}}{(2 \pi)^{2}} \sum_{b} n_{b \boldsymbol{k}}^{\mathrm{NO}} \Omega_{b \boldsymbol{k}}^{\mathrm{NO}}
$$

The result shown in Fig. 3(b) clearly demonstrates that lightinduced Berry-curvature has a nonzero contribution to the Hall conductivity. However, this contribution is much smaller.
Furthermore, by computing the dependence of this effect on the field strength, we find that it changes signs (see Appendix G).

Our modeling suggests that the recently observed lightinduced anomalous Hall effect in graphene results mainly from the asymmetric distribution of photocarriers in the topologically nontrivial Floquet-Bloch states while their Berry curvature contribution is clearly smaller. Nevertheless, it is striking that the theoretical Hall conductivity saturates on the order of $\sim 2 e^{2} / h$, which is consistent with the experimental observation [36], despite the strong population imbalance contribution (see also Appendix G). This may just be a coincidence, but the possibility of hidden connections to topological invariants should be investigated further. The anomalous velocity contribution from the Berry curvature of the Floquet-Bloch bands could become larger at higher laser frequencies or for more off-resonant driving, which might be realized in other material platforms $[48,49]$. The population imbalance effect can also be expected to play a role in quantum simulation experiments studying Floquet effects, such as of ultracold fermions in driven optical lattices [22,23,33], as well as in other driven quantum materials.

More broadly, these results highlight the importance of adopting a dressed-state picture in understanding the nonequilibrium electrical transport properties of optically driven solids. They also demonstrate the viability of Floquetengineering under experimentally realistic conditions, which opens the door to exploring a host of other exciting nonequilibrium quantum transport phenomena. For example, Floquet engineering of effective couplings in solids, such as dynamical Hubbard U, has recently been proposed as a means to significantly modify electronic states in correlated insulators [50-52] which can also induce nontrivial topology [53]. Future work could, for instance, explore the possibilities for light-controlled topological edge states [39,54] that are opened up by the present ultrafast-transport setup and complementary ultrafast real-space imaging techniques $[55,56]$.

This work was supported by the European Research Council (ERC-2015-AdG694097) and the Deutsche Forschungsgemeinschaft through the SFB 925. The Flatiron Institute is a division of the Simons Foundation. S.A.S. gratefully acknowledges the fellowship from the Alexander von Humboldt Foundation. M.A.S. acknowledges financial support by the DFG through the Emmy Noether program (SE 2558/2-1). P.T. acknowledges the received funding from the European Unions Horizon 2020 research and innovation program under the Marie Sklodowska-Curie Grant Agreement No 793609. M.N. acknowledges support from Stiftung der Deutschen Wirtschaft. L.M., A.R., and A.C. acknowledge support from the Cluster of Excellence Advanced Imaging of Matter (AIM).

\section{APPENDIX A: THEORETICAL MODELING}

Here, we describe the details of our theoretical modeling of electron dynamics in graphene under laser fields. The electron dynamics is described by the following quantum Liouville equation for the one-particle reduced density matrix:

$$
\frac{d}{d t} \rho_{\boldsymbol{K}(t)}(t)=\frac{\left[H_{\boldsymbol{K}(t)}, \rho_{\boldsymbol{K}(t)}\right]}{i \hbar}+\hat{D}_{\boldsymbol{K}(t)} \rho_{\boldsymbol{K}(t)}(t),
$$


with the Dirac Hamiltonian $H_{\boldsymbol{K}(t)}=\hbar v_{F} \tau_{z} \sigma_{x} K_{x}(t)+$ $\hbar v_{F} \sigma_{y} K_{y}(t)$ and an effective relaxation operator $\hat{D}_{\boldsymbol{K}(t)}$. Here, $v_{F}$ is the Fermi velocity, $\sigma_{x / y}$ is the Pauli matrix. The different chirality of the Dirac fermions at $K$ and $K^{\prime}$ points is given by $\tau_{z}= \pm 1$. In this paper, we employ a simple relaxation time approximation [44] for the relaxation operator $\hat{D}_{\boldsymbol{K}(t)}$. To construct the relaxation operator, we first represent the density matrix with instantaneous eigenstates of the Hamiltonian $H_{\boldsymbol{K}(t)}$ as

$$
\begin{aligned}
\rho_{\boldsymbol{K}(t)}(t): & =\left(\begin{array}{ll}
\rho_{v v, \boldsymbol{K}(t)}(t) & \rho_{v c, \boldsymbol{K}(t)}(t) \\
\rho_{c v, \boldsymbol{K}(t)}(t) & \rho_{c c, \boldsymbol{K}(t)}(t)
\end{array}\right) \\
& =\left(\begin{array}{ll}
\left\langle u_{v \boldsymbol{K}(t)}^{s}\left|\hat{\rho}_{\boldsymbol{K}(t)}(t)\right| u_{v \boldsymbol{K}(t)}^{s}\right\rangle & \left\langle u_{v \boldsymbol{K}(t)}^{s}\left|\hat{\rho}_{\boldsymbol{K}(t)}(t)\right| u_{c \boldsymbol{K}(t)}^{s}\right\rangle \\
\left\langle u_{c \boldsymbol{K}(t)}^{s}\left|\hat{\rho}_{\boldsymbol{K}(t)}(t)\right| u_{v \boldsymbol{K}(t)}^{s}\right\rangle & \left\langle u_{c \boldsymbol{K}(t)}^{s}\left|\hat{\rho}_{\boldsymbol{K}(t)}(t)\right| u_{c \boldsymbol{K}(t)}^{s}\right\rangle
\end{array}\right),
\end{aligned}
$$

where $\left|u_{b K(t)}^{s}\right\rangle$ is an instantaneous eigenstate of the Hamiltoinan, $H_{\boldsymbol{K}(t)}\left|u_{b \boldsymbol{K}(t)}^{s}\right\rangle=\epsilon_{b \boldsymbol{K}(t)}\left|u_{b \boldsymbol{K}(t)}^{s}\right\rangle$. Then, we construct the relaxation operator with the phenomenological relaxation times, $T_{1}$ and $T_{2}$, in the instantaneous eigenbasis expression as follows:

$$
\hat{D}_{\boldsymbol{K}(t)} \rho_{\boldsymbol{K}(t)}:=-\left(\begin{array}{ll}
\frac{\rho_{v v, \boldsymbol{K}(t)}(t)-\rho_{v K(t), \mu, T_{e}}^{m}}{T_{1}} & \frac{\rho_{v c, \boldsymbol{K}(t)}(t)}{T_{2}} \\
\frac{\rho_{c v, \boldsymbol{K}(t)}(t)}{T_{2}} & \frac{\rho_{c c, K(t)}(t)-\rho_{c \boldsymbol{K}(t), \mu, T_{e}}^{\mathrm{e}}}{T_{1}}
\end{array}\right),
$$

where $\rho_{b, \mu, T_{e}}^{\mathrm{eq}}$ is the Fermi-Dirac distribution,

$$
\rho_{b, \mu, T_{e}}^{\mathrm{eq}}=\frac{1}{e^{\left(\epsilon_{b K(t)}-\mu\right) / k_{B} T_{e}}+1},
$$

with the electron temperature $T_{e}$ and the chemical potential $\mu$. In this paper, we set the electron temperature to $80 \mathrm{~K}$ according to the experimental setup [36].

In this paper, the decoherence time $T_{2}$ is set to $20 \mathrm{fs}$ according to the electron-electron scattering time scale, while and the population relaxation time $T_{1}$ is set to $100 \mathrm{fs}$, according to the electron thermalization timescale [57-59]. However, as will be shown in the following section, the qualitative behaviors of the light-induced Hall effect do not much depend on the choice of the relaxation time.

\section{APPENDIX B: EVALUATION OF THE LIGHT-INDUCED HALL CONDUCTIVITY}

To investigate the light-induced Hall effect, we compute the electron dynamics under a circular midinfrared (MIR) laser pulse $\boldsymbol{E}_{\mathrm{MIR}}(t)$ and a static source-drain field $\boldsymbol{E}_{\mathrm{SD}}(t)$. In this paper, the pulse width of the MIR field is set to $1 \mathrm{ps}$, and the wavelength is set to $6.5 \mu \mathrm{m}$. The corresponding mean photon energy is about $\hbar \omega_{\mathrm{MIR}} \approx$ $190 \mathrm{meV}$. Furthermore, we assume that the MIR laser fields propagate along the $z$ axis, and the field polarizations are always on the $x-y$ plane. For the source-drain field, to prevent an artificial excitation, we employ the following smooth switching:

$$
\boldsymbol{E}_{\mathrm{SD}}(t)=E_{\mathrm{SD}} \boldsymbol{e}_{y} f\left(t / T_{\mathrm{Switch}}\right),
$$

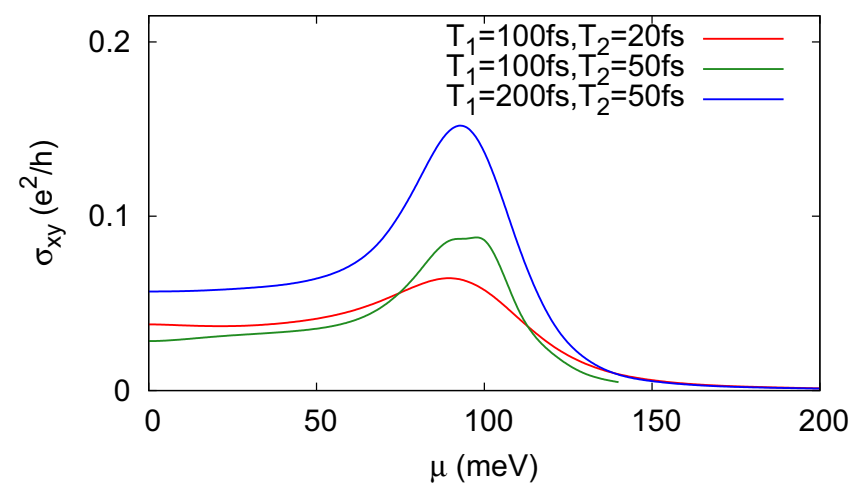

FIG. 4. The Hall conductivity $\sigma_{x y}$ as a function of chemical potential $\mu$ in the weak field regime. The results with different relaxation times, $T_{1}$ and $T_{2}$, are shown.

with the following switching function, $f(x)$ :

$$
f(x)= \begin{cases}1, & 1<x \\ 3 x^{2}-2 x^{3}, & 0<\leqslant 1 \\ 0, & \text { otherwise }\end{cases}
$$

In this paper, we set the source-drain direction to the $y$ direction, the source-drain field strength $E_{\mathrm{SD}}$ to $10^{4} \mathrm{MV} / \mathrm{m}$, and the switching time $T_{\text {switch }}$ to $20 \mathrm{fs}$.

To evaluate the Hall current, we compute the current along the $x$ direction under the presence of the circular MIR laser field and the source-drain field. Following the same analysis of the experiment [36], we compute two kinds of current, $J_{x}^{(\circlearrowright)}(t)$ and $J_{x}^{(\circlearrowleft)}(t): J_{x}^{(\circlearrowright)}(t)$ is induced by the right-handed circular laser $E_{\mathrm{MIR}}^{(\circlearrowright)}(t)$, while $J_{x}^{(\circlearrowleft)}(t)$ is induced by the lefthanded circular laser $E_{\mathrm{MIR}}^{(\circlearrowleft)}(t)$. Then, we define the difference of $J_{x}^{(\circlearrowright)}(t)$ and $J_{x}^{(\circlearrowleft)}(t)$ by $\Delta J_{x}(t)=\left[J_{x}^{(\circlearrowright)}(t)-J_{x}^{(\circlearrowleft)}(t)\right] / 2$. So far, the current $\Delta J_{x}(t)$ contains high-frequency components, which are not relevant for the transport property since the time-average of the high-frequency component becomes zero and there is no net charge transfer. To cleanly extract the transport property, we remove the irrelevant high-frequency component by the temporal average and define the theoretical Hall current as

$$
J_{H}(t)=\frac{1}{\sqrt{2 \pi \sigma^{2}}} \int_{-\infty}^{\infty} d t^{\prime} e^{-\frac{\left(t^{\prime}-t\right)^{2}}{2 \sigma^{2}}} \Delta J_{x}\left(t^{\prime}\right),
$$

where the width of the window $\sigma$ is set to $100 \mathrm{fs}$, which is substantially longer than the optical cycle but shorter than the pulse width. Furthermore, we define the Hall conductivity $\sigma_{x y}$ as the ratio of the peak Hall current $J_{H}\left(t_{\text {peak }}\right)$ and the sourcedrain field strength:

$$
\sigma_{x y}=\frac{J_{H}\left(t_{\text {peak }}\right)}{E_{\mathrm{SD}}} .
$$

\section{APPENDIX C: RELAXATION TIME DEPENDENCE}

Here, we explore the effect of the relaxation times, $T_{1}$ and $T_{2}$ in the light-induced Hall effect. Based on the above procedure, we compute the Hall conductivity $\sigma_{x y}$ as a function of chemical potential $\mu$. Figure 4 shows the computed Hall conductivity $\sigma_{x y}$ with the different relaxation times, $T_{1}$ and $T_{2}$, in the weak field regime, where the field strength of the 


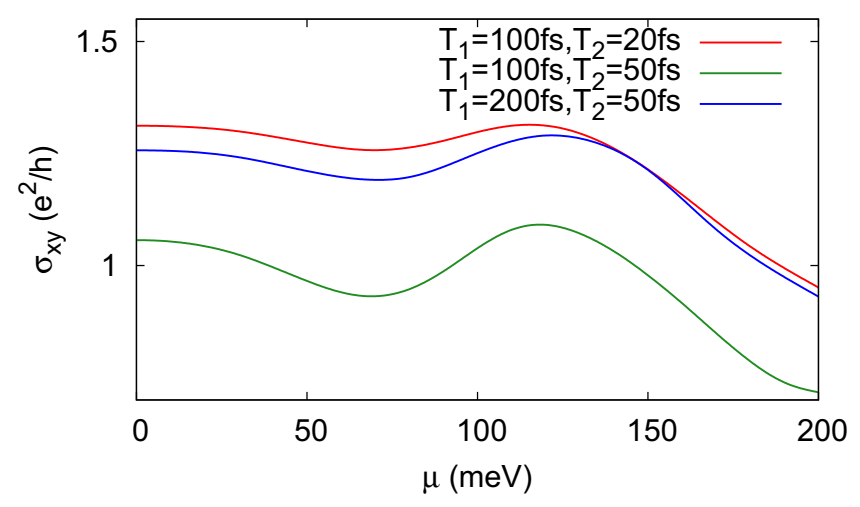

FIG. 5. The Hall conductivity $\sigma_{x y}$ as a function of chemical potential $\mu$ in the strong field regime. The results with different relaxation times, $T_{1}$ and $T_{2}$, are shown.

circular laser is set to $E_{\mathrm{MIR}}=1 \mathrm{MV} / \mathrm{m}$. The same results in the strong field regime $\left(E_{\mathrm{MIR}}=20 \mathrm{MV} / \mathrm{m}\right)$ are shown in Fig. 5. One can clearly confirm that the qualitative behaviors of the Hall conductivity do not depend on the choice of the relaxation time in both the weak and the strong field regimes.

\section{APPENDIX D: COMPARISON OF PULSED AND CONTINUOUS-WAVE LASER FIELDS}

Because the relaxation times, $T_{1}$ and $T_{2}$, are much shorter than the pulse width in this paper, the system is expected to realize a steady state due to the balance between the laser excitation and the relaxation. To confirm this fact, we compute the electron dynamics under a continuous-wave circular laser field instead of a laser pulse, and evaluate the Hall conductivity after the system reaches the steady state.

Figure 6 shows the light-induced Hall conductivities $\sigma_{x y}$ evaluated with a laser pulse and a continuous wave in the weak field regime, where the peak field strength is set to $1 \mathrm{MV} / \mathrm{m}$. The same comparison in the strong field regime $\left(E_{\mathrm{MIR}}=20 \mathrm{MV} / \mathrm{m}\right)$ is shown in Fig. 7. As seen from both figures, the Hall conductivity evaluated with a laser pulse is almost perfectly reproduced by that evaluated with the continuous-wave laser field. Therefore, we clearly confirmed

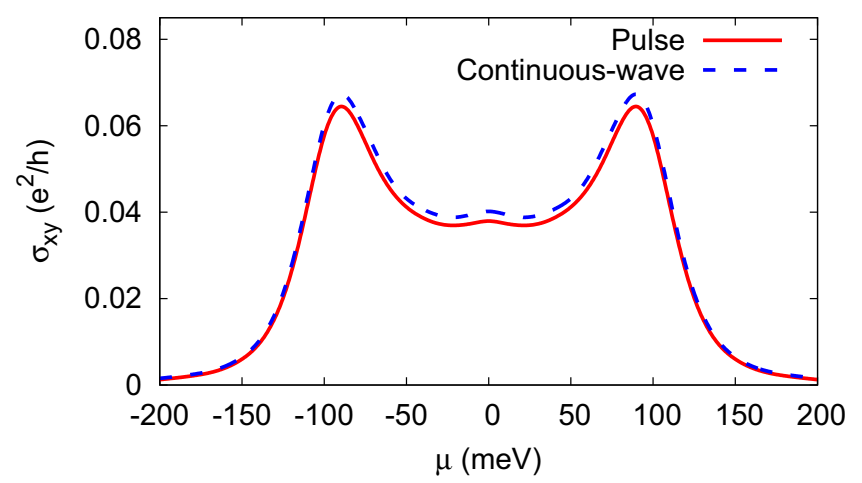

FIG. 6. The Hall conductivity $\sigma_{x y}$ as a function of chemical potential $\mu$ in the weak field regime. The results computed with the laser pulse (red solid) and the continuous-wave laser (blue dashed) are shown.

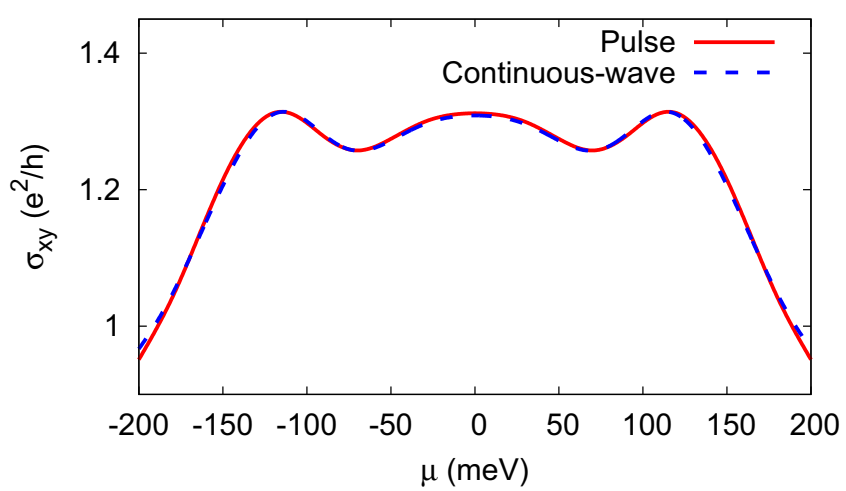

FIG. 7. The Hall conductivity $\sigma_{x y}$ as a function of chemical potential $\mu$ in the weak field regime. The results computed with the laser pulse (red solid) and the continuous-wave laser (blue dashed) are shown.

that the Hall conductivity evaluated by the laser pulse reflects the property of the steady state that is realized by the balance of the laser excitation and the relaxation.

\section{APPENDIX E: PERTURBATION ANALYSIS OF THE LIGHT-INDUCED HALL EFFECT IN THE DIRAC BAND}

To provide microscopic insight into the light-induced Hall effect in graphene, we apply a perturbative analysis. For simplicity, we ignore the relaxation effect, and thus, the system is described by the following Schrödinger equation instead of Eq. (A1):

$$
i \hbar \frac{d}{d t}\left|\psi_{k}(t)\right\rangle=H_{K(t)}\left|\psi_{k}(t)\right\rangle
$$

We perform the perturbative analysis based on the Houston state expansion [60,61],

$$
\begin{aligned}
\left|\psi_{\boldsymbol{k}}(t)\right\rangle= & c_{v \boldsymbol{k}}(t) e^{i \gamma_{v k}(t)} \exp \left[-\frac{i}{\hbar} \int_{0}^{t} d t^{\prime} \epsilon_{v \boldsymbol{K}\left(t^{\prime}\right)}\right]\left|u_{v \boldsymbol{K}(t)}^{s}\right\rangle \\
& +c_{c \boldsymbol{k}}(t) e^{i \gamma_{c k}(t)} \exp \left[-\frac{i}{\hbar} \int_{0}^{t} d t^{\prime} \epsilon_{c \boldsymbol{K}\left(t^{\prime}\right)}\right]\left|u_{c \boldsymbol{K}(t)}^{s}\right\rangle,
\end{aligned}
$$

where $\left|u s_{b \boldsymbol{K}(t)}\right\rangle$ is an eigenstate of the instantaneous Hamiltonian, $H_{\boldsymbol{K}(t)}, \epsilon_{b \boldsymbol{K}(t)}$ is its eigenvalue, $c_{b \boldsymbol{k}}(t)$ is an expansion coefficient, and $\gamma_{b k}(t)$ is a geometrical phase defined by

$$
\gamma_{b k}(t)=-i \int_{0}^{t} d t^{\prime}\left\langle u_{b K(t)}^{s}\left|\frac{d}{d t^{\prime}}\right| u_{b K(t)}^{s}\right\rangle
$$

For practical calculation, we assume the following forms for the the instantaneous eigenstates:

$$
\left|u_{v, \boldsymbol{K}(t)}^{s}\right\rangle=\frac{1}{\sqrt{2}}\left(\begin{array}{c}
1 \\
-\frac{\tau_{z} K_{x}(t)+i K_{y}(t)}{\sqrt{K_{x}^{2}(t)+K_{y}^{2}(t)}}
\end{array}\right)
$$

and

$$
\left|u_{c, \boldsymbol{K}(t)}^{s}\right\rangle=\frac{1}{\sqrt{2}}\left(\begin{array}{c}
1 \\
+\frac{\tau_{z} K_{x}(t)+i K_{y}(t)}{\sqrt{K_{x}^{2}(t)+K_{y}^{2}(t)}}
\end{array}\right) .
$$


Then, one can obtain the Schrödinger equation for the coefficient vectors,

$$
\begin{aligned}
i \hbar \frac{d}{d t} \boldsymbol{c}_{\boldsymbol{k}}(t)= & \frac{e}{2} \frac{\tau_{z}}{\boldsymbol{K}^{2}(t)}[\boldsymbol{K}(t) \times \boldsymbol{E}(t)]_{z} \\
& \times\left(\begin{array}{cc}
0 & e^{-\frac{2 \hbar \nu_{F}}{i \hbar} \int_{0}^{t} d t^{\prime}\left|\boldsymbol{K}\left(t^{\prime}\right)\right|} \\
e^{\frac{2 \hbar \nu_{F}}{i \hbar} \int_{0}^{t} d t^{\prime}\left|\boldsymbol{K}\left(t^{\prime}\right)\right|} & 0
\end{array}\right) \boldsymbol{c}_{\boldsymbol{k}}(t),
\end{aligned}
$$

where the coefficient vector $\boldsymbol{c}_{\boldsymbol{k}}(t)$ is defined as

$$
\boldsymbol{c}_{\boldsymbol{k}}(t)=\left(\begin{array}{c}
c_{c, \boldsymbol{k}}(t) \\
c_{v, k}(t)
\end{array}\right) .
$$

Then, we elucidate the nonlinear carrier-injection by the circular laser field and the source-drain field. For this purpose, we assume the following form for the electric field:

$$
\boldsymbol{E}(t)=\boldsymbol{e}_{x} E_{x}^{P}(t)+\boldsymbol{e}_{y} E_{x}^{P}(t)+\boldsymbol{e}_{x} E^{\mathrm{dc}},
$$

where $E_{x / y}^{P}$ denotes the pump electric field for $x / y$ direction, and $E^{\mathrm{dc}}$ denotes the static electric field for $x$ direction.

Thanks to the structure of the Hamiltonian in Eq. (E6), the coefficient vector, $\boldsymbol{c}_{\boldsymbol{k}}(t)$, can be accurately evaluated up to the second-order of the electric fields as

$$
\begin{aligned}
\boldsymbol{c}_{\boldsymbol{k}}(t)= & \frac{1}{i \hbar} \int_{0}^{t} d t^{\prime} \frac{e}{2} \frac{\tau_{z}}{\boldsymbol{K}^{2}\left(t^{\prime}\right)}\left[\boldsymbol{K}\left(t^{\prime}\right) \times \boldsymbol{E}\left(t^{\prime}\right)\right]_{z} \\
& \times\left(\begin{array}{cc}
0 & e^{-\frac{2 \hbar \hbar}{i \hbar} \int_{0}^{t^{\prime}} d t^{\prime \prime}\left|\boldsymbol{K}\left(t^{\prime \prime}\right)\right|} \\
e^{\frac{2 \hbar v}{i \hbar} \int_{0}^{t^{\prime}} d t^{\prime \prime}\left|\boldsymbol{K}\left(t^{\prime \prime}\right)\right|} & 0
\end{array}\right)\left(\begin{array}{l}
0 \\
1
\end{array}\right) .
\end{aligned}
$$

The conduction component can be written as

$$
\begin{aligned}
c_{+, k}(t \rightarrow \infty)= & \frac{1}{i \hbar} \int_{0}^{\infty} d t^{\prime} \frac{e}{2} \frac{\tau_{z}}{\boldsymbol{K}^{2}\left(t^{\prime}\right)}\left[\boldsymbol{K}\left(t^{\prime}\right) \times \boldsymbol{E}\left(t^{\prime}\right)\right]_{z} \\
& \times e^{-\frac{2 \hbar v}{i \hbar} \int_{0}^{t^{\prime}} d t^{\prime \prime}\left|\boldsymbol{K}\left(t^{\prime \prime}\right)\right|} .
\end{aligned}
$$

For simplicity, we assume that (i) the Fourier transform of the pump electric field $\left|\tilde{E}_{x / y}^{P}(\omega)\right|$ is localized in the frequency domain around $\hbar \omega=\hbar|\boldsymbol{k}| v_{F}$ and (ii) $\boldsymbol{k}$ is on $y$ axis; $k_{x}=0$. Ignoring the third and higher order contributions in Eq. (E11), we obtain

$$
\begin{aligned}
c_{+, k}(t \rightarrow \infty)= & -\frac{\tau_{z}}{i \hbar} \frac{e}{2} \frac{k_{y}}{k^{2}} \int_{0}^{\infty} d t^{\prime} E_{x}^{P}\left(t^{\prime}\right) e^{2 i v k t^{\prime}} \\
& +\frac{\tau_{z}}{i \hbar} \frac{e}{2} \frac{e}{\hbar} \frac{1}{k^{2}} \int_{0}^{\infty} d t^{\prime} \frac{A^{\mathrm{dc}}\left(t^{\prime}\right)}{c} E_{y}^{P}\left(t^{\prime}\right) e^{2 i v k t^{\prime}} \\
& +\frac{\tau_{z}}{i \hbar} \frac{e}{2} \frac{e}{\hbar} \frac{1}{k^{2}} E_{x}^{\mathrm{dc}} \int_{0}^{\infty} d t^{\prime} \frac{A_{y}^{P}\left(t^{\prime}\right)}{c} e^{2 i v k t^{\prime}} \\
& -\frac{\tau_{z}}{i \hbar} \frac{e}{2} \frac{e}{\hbar} \frac{2 i v}{k} E_{x}^{\mathrm{dc}} e^{2 i v k t^{\prime}} \int_{0}^{t^{\prime}} d t^{\prime \prime} \frac{A^{P}\left(t^{\prime \prime}\right)}{c}
\end{aligned}
$$

where $A_{x / y}^{P}$ and $A^{\mathrm{dc}}$ are amplitude of the vector potentials corresponding to the electric fields $E_{x / y}^{P}$ and $E^{\mathrm{dc}}$, respectively.

On the right-hand side of Eq. (E11), the first term corresponds to the one photon absorption process by the pump light with the first-order perturbation. The second term corresponds to the nonlinear excitation due to the coupling of the pumpinduced interband transition and the dc-field-induced intraband acceleration. The third and the last terms correspond to the nonlinear coupling of the pump-induced intraband acceleration and the dc-field-induced interband transition. Therefore, we may classify the first term as the direct resonant excitation and the rest of the terms as the indirect resonant excitation assisted by the source-drain field.

One may further proceed with the evaluation as

$$
\begin{aligned}
c_{+, k}(t \rightarrow \infty)= & -\frac{\tau_{z}}{i \hbar} \frac{e}{2} \frac{k_{y}}{k^{2}} \tilde{E}_{x}^{P}\left(2 v k_{y}\right) \\
& +\left.\frac{\tau_{z}}{\hbar} \frac{e}{2} \frac{e}{\hbar} \frac{1}{k^{2}} E_{x}^{d c} \frac{\partial}{\partial \omega} \tilde{E}_{y}^{P}(\omega)\right|_{\omega=2 v k} \\
& -\frac{\tau_{z}}{\hbar} \frac{e}{2} \frac{e}{\hbar} \frac{1}{k^{2}} \frac{1}{v k} E_{x}^{d c} \tilde{E}_{y}^{P}(2 v k) .
\end{aligned}
$$

Here, as explained above, the first term corresponds to the resonant excitation by the pump pulse [the first term of Eq. (E12)], while the other terms correspond to the nonlinear photocarrier injection assisted by the static electric field.

Assuming perfect circular pump laser, $\tilde{E}^{P}(\omega) \equiv \tilde{E}_{x}^{P}(\omega)=$ $\pm i \tilde{E}_{y}^{P}(\omega)$, the injected population on $y$ axis can be evaluated up to the first order of $E^{\mathrm{dc}}$ as

$$
\begin{aligned}
n_{c}\left(k_{y}, k_{x}=0\right)= & \left|c_{+, k}(t \rightarrow \infty)\right|^{2} \\
\approx & \left(\frac{1}{\hbar} \frac{e}{2}\right)^{2} \frac{1}{k^{2}}\left|\tilde{E}^{P}(\omega=2 v k)\right|^{2} \\
& \left.\mp\left(\frac{1}{\hbar} \frac{e}{2}\right)^{2} \frac{e}{\hbar} \frac{k_{y}}{k^{4}} E_{x}^{d c} \frac{\partial}{\partial \omega}\left|\tilde{E}^{P}(\omega)\right|^{2}\right|_{\omega=2 v k} \\
& \pm\left(\frac{1}{\hbar} \frac{e}{2}\right)^{2} \frac{e}{\hbar} \frac{k_{y}}{k^{4}} \frac{2}{v k} E_{x}^{d c}\left|\tilde{E}^{P}(\omega=2 v k)\right|^{2} .
\end{aligned}
$$

Here, the upper sign corresponds to the right-handed circular pump, while the lower sign corresponds to the left handed. Therefore, the population difference between the right- and left-handed circular pump becomes

$$
\begin{aligned}
\Delta n_{c}\left(k_{y}, k_{x}=0\right)= & n_{c}^{(\circlearrowright)}\left(k_{y}, k_{x}=0\right)-n_{c}^{(\circlearrowleft)}\left(k_{y}, k_{x}=0\right) \\
= & -\left.2\left(\frac{1}{\hbar} \frac{e}{2}\right)^{2} \frac{e}{\hbar} \frac{k_{y}}{k^{4}} E_{x}^{d c} \frac{\partial}{\partial \omega}\left|\tilde{E}^{P}(\omega)\right|^{2}\right|_{\omega=2 v k} \\
& +4\left(\frac{1}{\hbar} \frac{e}{2}\right)^{2} \frac{e}{\hbar} \frac{k_{y}}{k^{4}} \frac{1}{v k} E_{x}^{d c}\left|\tilde{E}^{P}(\omega=2 v k)\right|^{2} .
\end{aligned}
$$

One sees that the population difference of Eq. (E14) breaks the symmetry for $k_{y}$ direction. Therefore, the population imbalance is clearly formed along the $y$ direction under the static field along the $x$ direction. Note that the population imbalance is formed by the interference between the direct resonant excitation [the first term of Eq. (E12)] and the nonlinear photocarrier injection assisted by the presence of the sourcedrain field [the other terms of Eq. (E12)].

Because the population distribution has a direct contribution to the intraband component of current, the population imbalance along the $y$ direction in the momentum space under 
the static field along the $x$ direction results in the net Hall current.

\section{Tilting of Dirac bands under a static field}

To understand the role of the static electric field in the formation of the population imbalance in Eq. (E14), we elucidate the modification of the electronic structure induced purely by the static electric field. For this purpose, we reconsider the time-dependent Schrödinger Eq. (E1) with the following ansatz:

$$
\left|\psi_{\boldsymbol{k}}(t)\right\rangle=d_{v, \boldsymbol{k}}(t)\left|u_{v \boldsymbol{K}(t)}^{s}\right\rangle+d_{c, \boldsymbol{k}}(t)\left|u_{c \boldsymbol{K}(t)}^{s}\right\rangle .
$$

The coefficient vector $\boldsymbol{d}_{k}(t)$ satisfies the following Schrödinger equation under a static electric field $\boldsymbol{E}_{0}$ :

$$
\begin{aligned}
i \hbar \frac{d}{d t} \boldsymbol{d}_{k}(t)= & \hbar v|\boldsymbol{K}(t)|\left(\begin{array}{cc}
1 & 0 \\
0 & -1
\end{array}\right) \boldsymbol{d}_{\boldsymbol{k}}(t) \\
& +\frac{\tau_{z}}{2} \frac{e}{K^{2}}\left[\boldsymbol{K} \times \boldsymbol{E}_{0}\right]_{z}\left(\begin{array}{cc}
-1 & 1 \\
1 & -1
\end{array}\right) \boldsymbol{d}_{\boldsymbol{k}}(t) .
\end{aligned}
$$

Then, we evaluate the eigenvalues of the above effective Hamiltonian and obtain

$$
\begin{aligned}
\tilde{\epsilon}_{c / v, \boldsymbol{K}}(t)= & \pm \sqrt{(\hbar v|\boldsymbol{K}(t)|)^{2}+\left(\frac{\tau_{z} e}{2 K^{2}}\left[\boldsymbol{K} \times \boldsymbol{E}_{0}\right]_{z}\right)^{2}} \\
& -\frac{\tau_{z} e}{2 K^{2}}\left[\boldsymbol{K} \times \boldsymbol{E}_{0}\right]_{z} .
\end{aligned}
$$

In the weak field limit, the eigenvalues can be approximated by

$$
\tilde{\epsilon}_{c / v, \boldsymbol{K}(t)}= \pm \hbar v_{F}|\boldsymbol{K}(t)|-\frac{\tau_{z} e}{2 K^{2}}\left[\boldsymbol{K} \times \boldsymbol{E}_{0}\right]_{z} .
$$

The first term is nothing but the energy of the bare Dirac band, and the second term is the modification due to the applied static electric field. One sees that the modification induces the distorted tilt to the bare Dirac band. Therefore, we can conclude that the static electric field assists the nonlinear photocarrier injection with the pump pulse by tilting the Dirac band.

\section{APPENDIX F: PERTURBATION ANALYSIS OF THE LIGHT-INDUCED HALL EFFECT IN A PARABOLIC TWO-BAND SEMICONDUCTOR}

In this section, we investigate the light-induced Hall effect in a simple parabolic two-band model to demonstrate that the population effect discussed in Appendix E is a rather general mechanism.

\section{Parabolic two-band model}

First, we construct a parabolic two-band model. For this purpose, we start from the following one-body Schrödinger equation:

$$
\begin{aligned}
i \hbar \frac{\partial}{\partial t} u_{b \boldsymbol{k}}(\boldsymbol{r}, t) & =\left[\frac{1}{2 m}\left\{\boldsymbol{p}+\hbar \boldsymbol{k}+\frac{e}{c} \boldsymbol{A}(t)\right\}^{2}+v(\boldsymbol{r})\right] u_{b \boldsymbol{k}}(\boldsymbol{r}, t) \\
& =\hat{h}_{\boldsymbol{K}(t)} u_{b \boldsymbol{k}}(\boldsymbol{r}, t),
\end{aligned}
$$

where $u_{b k}(\boldsymbol{r}, t)$ is a time-dependent Bloch state, and $v(\boldsymbol{r})$ is a one-body potential that has the same periodicity as the crystal. Here, $b$ denotes a band index, while $\boldsymbol{k}$ denotes the Bloch wave number. We note that the crystal momentum is shifted by the vector potential as $\boldsymbol{K}(t)=\boldsymbol{k}+e \boldsymbol{A}(t) / \hbar c$.

Then, we introduce the Houston state $[60,61]$ as a solution of the Schrödinger Eq. (F1) in the adiabatic limit,

$$
u_{b k}^{H}(\boldsymbol{r}, t)=\exp \left[-\frac{i}{\hbar} \int_{0}^{t} d t^{\prime} \epsilon_{b \boldsymbol{K}\left(t^{\prime}\right)}\right] u_{b \boldsymbol{K}(t)}^{S},
$$

where $\epsilon_{b \boldsymbol{K}(t)}$ and $u_{b \boldsymbol{K}(t)}^{S}(\boldsymbol{r})$ are an eigenvalue and the eigenstate of the instantaneous Hamiltonian, $\hat{h}_{\boldsymbol{K}(t)}$, respectively:

$$
\hat{h}_{\boldsymbol{K}(t)} u_{b \boldsymbol{K}(t)}^{S}(\boldsymbol{r})=\epsilon_{b \boldsymbol{K}(t)} u_{b \boldsymbol{K}(t)}^{S}(\boldsymbol{r}) .
$$

Here, we assume the following condition for the diagonal element of the $k$-derivative operator for all bands $b$ and all $\boldsymbol{k}$ :

$$
\int_{\Omega} d \boldsymbol{r} u_{b \boldsymbol{k}}^{S, *}(\boldsymbol{r}) \frac{\partial}{\partial \boldsymbol{k}} u_{b \boldsymbol{k}}^{S}(\boldsymbol{r})=0
$$

This condition guarantees that there is no Berry curvature at all $\boldsymbol{k}$.

To construct a two-band model, we assume that the wave function at each $k$-point can be expanded by only two Houston states; one representing a valance, and the other a conduction state:

$$
u_{k}(\boldsymbol{r}, t)=c_{v k}(t) u_{v k}^{H}(\boldsymbol{r}, t)+c_{c k}(t) u_{c k}^{H}(\boldsymbol{r}, t) .
$$

Inserting Eq. (F5) into Eq. (F1), one can derive an equation of motion for the coefficients $c_{v \boldsymbol{k}}(t)$ and $c_{c k}(t)$,

$$
i \hbar \frac{d}{d t}\left(\begin{array}{l}
c_{v k}(t) \\
c_{c k}(t)
\end{array}\right)=\left(\begin{array}{cc}
0 & h_{v c, \boldsymbol{k}}(t) \\
h_{v c, \boldsymbol{k}}^{*}(t) & 0
\end{array}\right)\left(\begin{array}{l}
c_{v \boldsymbol{k}}(t) \\
c_{c \boldsymbol{k}}(t)
\end{array}\right)
$$

where the off-diagonal matrix element is given by

$$
h_{v c, \boldsymbol{k}}(t)=-\frac{i \boldsymbol{p}_{v c, \boldsymbol{K}(t)} \cdot \boldsymbol{E}(t)}{\epsilon_{v, \boldsymbol{K}(t)}-\epsilon_{c, \boldsymbol{K}(t)}} \frac{e \hbar}{m} e^{\frac{1}{i \hbar} t^{t} d t^{\prime}\left\{\epsilon_{c, \boldsymbol{K}\left(t^{\prime}\right)}-\epsilon_{v, \boldsymbol{K}\left(t^{\prime}\right)}\right\}}
$$

and

$$
\boldsymbol{p}_{v c, \boldsymbol{K}(t)}=\int_{\Omega} d \boldsymbol{r} u_{v \boldsymbol{K}(t)}^{S, *}(\boldsymbol{r}) \boldsymbol{p} u_{c \boldsymbol{K}(t)}^{S}(\boldsymbol{r}),
$$

where $\Omega$ is the volume of the unit cell. Note that Eq. (F6) is nothing but the Houston state expansion of the Schrödinger equation $[60,61]$ with only two Houston states.

To further simplify the model, we approximate the electronic structure by the parabolic bands as

$$
\begin{aligned}
& \epsilon_{v, k}=-\frac{\hbar^{2} \boldsymbol{k}^{2}}{2 m_{v}}, \\
& \epsilon_{c, \boldsymbol{k}}=\epsilon_{g}+\frac{\hbar^{2} \boldsymbol{k}^{2}}{2 m_{c}},
\end{aligned}
$$

where $\epsilon_{g}$ is the band gap, and $m_{v}$ and $m_{c}$ are the effective masses for valence and conduction bands, respectively. Here, we also define the reduced mass $\mu^{-1}=m_{v}^{-1}+m_{c}^{-1}$. 


\section{Perturbation analysis for light-induced Hall current}

Here, we investigate the light-induced Hall current in the parabolic two-band model with the perturbation theory. We set the initial wave function to the valence state. Thanks to the structure of the Schrödinger equation (F6), the timedependent conduction coefficient, $c_{c k}(t)$, can be accurately described by the following expression up to the second order of the electric field $\boldsymbol{E}$ :

$$
\begin{aligned}
c_{c k}(t) & =\frac{1}{i \hbar} \int_{0}^{t} d t^{\prime} h_{v c, \boldsymbol{k}}^{*}\left(t^{\prime}\right) \\
& =-\frac{1}{i \hbar} \int_{0}^{t} d t^{\prime} \frac{i \boldsymbol{p}_{c v, \boldsymbol{K}\left(t^{\prime}\right)} \cdot \boldsymbol{E}\left(t^{\prime}\right)}{\epsilon_{c, \boldsymbol{K}\left(t^{\prime}\right)}-\epsilon_{v, \boldsymbol{K}\left(t^{\prime}\right)}} \frac{e \hbar}{m} e^{\frac{i}{\hbar} \int^{t^{\prime}} d t^{\prime \prime}\left\{\epsilon_{c, \boldsymbol{K}\left(t^{\prime \prime}\right)}-\epsilon_{v, \boldsymbol{K}\left(t^{\prime \prime}\right)}\right\}} \\
& =-\frac{1}{i \hbar} \int_{0}^{t} d t^{\prime} \frac{i \boldsymbol{p}_{c v, \boldsymbol{K}\left(t^{\prime}\right)} \cdot \boldsymbol{E}\left(t^{\prime}\right)}{\Delta \epsilon_{\boldsymbol{K}\left(t^{\prime}\right)}} \frac{e \hbar}{m} e^{\frac{i}{\hbar} t^{t^{\prime}} d t^{\prime \prime}\left\{\Delta \epsilon_{K\left(t^{\prime \prime}\right)}\right\}},
\end{aligned}
$$

where $\Delta \epsilon_{\boldsymbol{K}\left(t^{\prime \prime}\right)}$ denotes the energy gap, $\epsilon_{c, \boldsymbol{K}\left(t^{\prime \prime}\right)}-\epsilon_{v, \boldsymbol{K}\left(t^{\prime \prime}\right)}$.

For simplicity, we neglect the time dependence of the dipole matrix element, $\boldsymbol{p}_{c v, \boldsymbol{K}(t)} / \Delta \epsilon_{\boldsymbol{K}(t)}$, and we can simplicity Eq. (F11) as

$$
c_{c k}(t)=-\frac{1}{i \hbar} \int_{0}^{t} d t^{\prime} \frac{i \boldsymbol{p}_{c v, \boldsymbol{k}} \cdot \boldsymbol{E}\left(t^{\prime}\right)}{\Delta \epsilon_{\boldsymbol{k}}} \frac{e \hbar}{m} e^{\frac{i}{\hbar} \int^{t^{\prime}} d t^{\prime \prime}\left\{\Delta \epsilon_{\boldsymbol{K}\left(t^{\prime \prime}\right)}\right\}} .
$$

Furthermore, we expand the contribution from the dynamical phase factor up to the first order of the electric field, and we obtain

$$
\begin{aligned}
c_{c k}(t)= & -\frac{1}{i \hbar} \int_{0}^{t} d t^{\prime} \frac{i \boldsymbol{p}_{c v, \boldsymbol{k}} \cdot \boldsymbol{E}\left(t^{\prime}\right)}{\Delta \epsilon_{\boldsymbol{k}}} \frac{e \hbar}{m} e^{\frac{i}{\hbar} \Delta \epsilon_{k} t^{\prime}} \\
& \times\left[1+\frac{i}{\hbar} \int_{0}^{t^{\prime}} d t^{\prime \prime} \frac{\hbar \boldsymbol{k}}{\mu} \cdot \frac{e}{c} \boldsymbol{A}\left(t^{\prime \prime}\right)\right]
\end{aligned}
$$

To proceed with the analysis, we assume the following form for the applied electric field:

$$
\boldsymbol{E}(t)=\boldsymbol{e}_{x} E_{x}^{P}(t)+\boldsymbol{e}_{y} E_{y}^{P}(t)+\boldsymbol{e}_{x} E_{x}^{d c} \Theta(t)
$$

where $E_{x / y}^{P}(t)$ is the pump electric field for $x / y$-direction, while $E_{x}^{\mathrm{dc}}$ is the static electric field for $x$ direction. We further assume that the photon energy of the pump pulse is localized around the vertical gap $\Delta \epsilon_{\boldsymbol{k}}$ at $\boldsymbol{k}$.

For simplicity, here we only consider the excitation on $k_{y}$ axis, assuming $k_{x}=0$. Then, one can evaluate Eq. (F13) as

$$
\begin{aligned}
c_{c k}(t)= & -\frac{1}{i \hbar} \int_{0}^{t} d t^{\prime} \frac{i \boldsymbol{p}_{c v, \boldsymbol{k}} \cdot \boldsymbol{E}^{P}\left(t^{\prime}\right)}{\Delta \epsilon_{\boldsymbol{k}}} \frac{e \hbar}{m} e^{\frac{i}{\hbar} \Delta \epsilon_{k} t^{\prime}} \\
& -\frac{1}{i \hbar} \int_{0}^{t} d t^{\prime} \frac{i p_{c v, \boldsymbol{k}, x} E_{x}^{d c}}{\Delta \epsilon_{k}} \frac{e \hbar}{m} e^{\frac{i}{\hbar} \Delta \epsilon_{k} t^{\prime}} \\
& \times \frac{i}{\hbar} \int_{0}^{t^{\prime}} d t^{\prime \prime} \frac{\hbar k_{y}}{\mu} \cdot \frac{e}{c} A_{y}^{P}\left(t^{\prime \prime}\right) .
\end{aligned}
$$

Therefore, the conduction coefficient after infinite time can be evaluated as

$$
\begin{aligned}
c_{c \boldsymbol{k}}(t \rightarrow \infty)= & -\frac{1}{i \hbar} \frac{i \boldsymbol{p}_{c v, \boldsymbol{k}} \cdot \tilde{\boldsymbol{E}}^{P}\left(\omega=\Delta \epsilon_{\boldsymbol{k}} / \hbar\right)}{\Delta \epsilon_{\boldsymbol{k}}} \frac{e \hbar}{m} \\
& -\frac{1}{i \hbar} \frac{i p_{c v, \boldsymbol{k}, x} E_{x}^{d c}}{\Delta \epsilon_{\boldsymbol{k}}} \frac{e \hbar}{m} \frac{i}{\hbar} \frac{e \hbar k_{y}}{\mu} \frac{\tilde{E}_{y}^{P}\left(\omega=\Delta \epsilon_{\boldsymbol{k}} / \hbar\right)}{\Delta \epsilon_{\boldsymbol{k}}^{2}} .
\end{aligned}
$$

Here, the first term of Eq. (F16) corresponds to the one photon absorption process with the first-order perturbation theory, while the second term corresponds to the nonlinear photocarrier injection under the presence of the static electric field.

Using the derived coefficients in Eq. (F16), the conduction population can be expressed as

$$
\begin{aligned}
n_{c k}= & \left|c_{c \boldsymbol{k}}(t \rightarrow \infty)\right|^{2} \\
= & \frac{e^{2}}{m^{2}} \frac{1}{\Delta \epsilon_{\boldsymbol{k}}^{2}}\left|\boldsymbol{p}_{c v, \boldsymbol{k}} \cdot \tilde{\boldsymbol{E}}^{P}\left(\omega=\Delta \epsilon_{\boldsymbol{k}} / \hbar\right)\right|^{2} \\
& +i \frac{e^{2}}{m^{2}} \frac{1}{\Delta \epsilon_{\boldsymbol{k}}^{4}} \frac{e k_{y}}{\mu} p_{c v, \boldsymbol{k}, x} E_{x}^{d c} \\
& \times\left(\boldsymbol{p}_{c v, \boldsymbol{k}} \cdot \tilde{\boldsymbol{E}}^{P}\left(\omega=\Delta \epsilon_{\boldsymbol{k}} / \hbar\right)\right)^{*} \tilde{E}_{y}^{P}\left(\omega=\Delta \epsilon_{\boldsymbol{k}} / \hbar\right)+\text { c.c. }
\end{aligned}
$$

Assuming perfect circularly polarized light for the pump, $\tilde{E}^{P}(\omega) \equiv \tilde{E}_{x}^{P}(\omega)= \pm i \tilde{E}_{y}^{P}(\omega)$, we evaluate the injectedpopulation difference by the right-handed and the left-handed circular light as

$$
\begin{aligned}
\Delta n_{c, \boldsymbol{k}}= & n_{c \boldsymbol{k}}^{(\circlearrowright)}-n_{c \boldsymbol{k}}^{(\circlearrowleft)}=-4 \frac{e^{2}}{m^{2}} \frac{1}{\Delta \epsilon_{k}^{4}} \frac{e k_{y}}{\mu} E_{x}^{d c}\left|p_{c v, \boldsymbol{k}, x}\right|^{2} \\
& \times\left|\tilde{E}^{P}\left(\omega=\Delta \epsilon_{\boldsymbol{k}} / \hbar\right)\right|^{2} .
\end{aligned}
$$

If we assume the time-reversal symmetry for the groundstate Hamiltonian, the transition momentum holds:

$$
\left|p_{c v, \boldsymbol{k}, x}\right|=\left|p_{c v,-\boldsymbol{k}, x}\right| .
$$

Therefore, Eq. (F18) indicates that there can be population imbalance in $k$-space under circularly polarized light and static voltage. Thus, we can conclude that the light-induced Hall current can be induced even in a topologically trivial (conventional) insulator/semiconductor with the population imbalance mechanism.

\section{APPENDIX G: FIELD STRENGTH SCALING}

Here, we investigate the field strength dependence of the light-induced Hall effect. Figure 8 shows the computed Hall conductivity for different field strengths of applied circular laser pulses. The red line shows the total conductivity, while the blue line shows the Berry curvature contribution computed by Eq. (4) in the main text. As seen from the figure, the total Hall conductivity monotonically increases with the applied field strength, while the Berry curvature contribution shows rather complex behaviors with the sign change. The complex behavior can be understood as the high nonlinear population transfer among different Floquet states because the different 


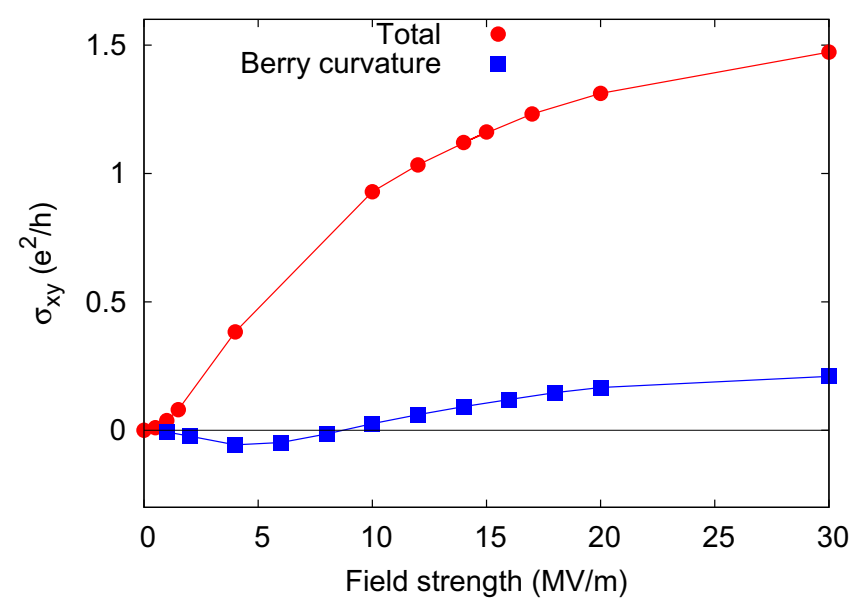

FIG. 8. The Hall conductivity $\sigma_{x y}$ as a function of applied field strength. The total conductivity is shown as the red line, while the topological contribution is shown as the blue line.

Floquet states have different contribution to the Hall effects, and those contributions are strongly canceled by each other due to the significant population transfer. Since the population transfer is highly nonlinear and nontrivial in the strong field regime, the resulting Hall conductivity shows the complex behaviors.

\section{APPENDIX H: FLOQUET FIDELITY}

To clarify the relation between the natural orbitals and the Floquet states under a continuous-wave laser field, we introduce Floquet fidelity, $S_{k}$, as a measure of the similarity of the two kinds of states.

Under a continuous-wave laser field, the system that obeys Eq. (A1) reaches a steady state due to the balance of the laser excitation and the relaxation. Such steady states can be described by a time-periodic density matrix,

$$
\rho_{\boldsymbol{K}(t)}(t)=\rho_{\boldsymbol{K}(t)}(t+T),
$$

where $T$ is an optical cycle of the driving laser field. Therefore, the natural orbitals $\left|u_{k}^{\mathrm{NO}}(t)\right\rangle$, which are eigenvectors of the density matrix, also have the same time periodicity $T$.

Floquet states $\left|u_{b k}^{F}(t)\right\rangle$ also have the same time periodicity as $\left|u_{b \boldsymbol{k}}^{F}(t+T)\right\rangle=\left|u_{b \boldsymbol{k}}^{F}(t)\right\rangle$ and they satisfy the time-dependent
Schrödinger Eq. (E1) under the periodic driving field as

$$
i \hbar \frac{d}{d t}\left|\psi_{b k}^{F}(t)\right\rangle=H_{\boldsymbol{K}(t)}\left|\psi_{b k}^{F}(t)\right\rangle
$$

with $\left|\psi_{b k}^{F}(t)\right\rangle=e^{-i \epsilon_{b k}^{F} t / \hbar}\left|u_{b k}^{F}(t)\right\rangle$, where $\epsilon_{b k}^{F}$ is the so-called Floquet quasienergy.

To define the similarity of the natural orbitals and the Floquet states, we first consider the cycle-average quantum fidelity $F_{i j k}$, which is equivalent to the squared overlap:

$$
F_{i j k}=\frac{1}{T} \int_{0}^{T} d t\left|\left\langle u_{i k}^{\mathrm{NO}}(t) \mid u_{j k}^{F}(t)\right\rangle\right|^{2} .
$$

Then, we define the Floquet fidelity $S_{k}$ as the absolute value of the determinant of the fidelity matrix $F_{k}$ that consists of $F_{i j k}$ as an element:

$$
S_{k}=\left|\operatorname{det} F_{k}\right|
$$

The Floquet fidelity $S_{k}$ satisfies

$$
0 \leqslant S_{k} \leqslant 1,
$$

and $S_{k}=1$ only if all the natural orbitals are identical to the Floquet states of the system. In contrast, if the natural orbitals are fully delocalized on the Floquet basis, the Floquet fidelity $S_{\boldsymbol{k}}$ becomes zero. For example, if the two natural orbitals are identical to the two Floquet states,

$$
\begin{aligned}
& u_{v k}^{N O}(t)=u_{v k}^{F}(t), \\
& u_{c k}^{N O}(t)=u_{c k}^{F}(t),
\end{aligned}
$$

the Floquet fidelity becomes one as

$$
S_{k}=\left|\operatorname{det}\left(\begin{array}{ll}
1 & 0 \\
0 & 1
\end{array}\right)\right|=1 .
$$

On the other hand, if the two natural orbitals are fully delocalized on the Floquet basis such as

$$
\begin{aligned}
& u_{v \boldsymbol{k}}^{N O}(t)=\frac{1}{\sqrt{2}}\left[u_{v \boldsymbol{k}}^{F}(t)+u_{c k}^{F}(t)\right], \\
& u_{c k}^{N O}(t)=\frac{1}{\sqrt{2}}\left[u_{v k}^{F}(t)-u_{c k}^{F}(t)\right],
\end{aligned}
$$

the Floquet fidelity becomes zero as

$$
S_{k}=\left|\operatorname{det}\left(\begin{array}{ll}
1 / 2 & 1 / 2 \\
1 / 2 & 1 / 2
\end{array}\right)\right|=0
$$

[1] D. N. Basov, R. D. Averitt, and D. Hsieh, Nat. Mater. 16, 1077 (2017).

[2] F. Krausz and M. I. Stockman, Nat. Photon. 8, 205 (2014).

[3] D. Fausti, R. I. Tobey, N. Dean, S. Kaiser, A. Dienst, M. C. Hoffmann, S. Pyon, T. Takayama, H. Takagi, and A. Cavalleri, Science 331, 189 (2011).

[4] M. Mitrano, A. Cantaluppi, D. Nicoletti, S. Kaiser, A. Perucchi, S. Lupi, P. Di Pietro, D. Pontiroli, M. Riccò, S. R. Clark, D. Jaksch, and A. Cavalleri, Nature 530, 461 (2016).
[5] M. Fiebig, K. Miyano, Y. Tomioka, and Y. Tokura, Science 280, 1925 (1998).

[6] A. Cavalleri, C. Tóth, C. W. Siders, J. A. Squier, F. Ráksi, P. Forget, and J. C. Kieffer, Phys. Rev. Lett. 87, 237401 (2001).

[7] M. Rini, A. Cavalleri, R. W. Schoenlein, R. López, L. C. Feldman, R. F. Haglund, L. A. Boatner, and T. E. Haynes, Opt. Lett. 30, 558 (2005).

[8] M. Liu, H. Y. Hwang, H. Tao, A. C. Strikwerda, K. Fan, G. R. Keiser, A. J. Sternbach, K. G. West, S. Kittiwatanakul, J. Lu, 
S. A. Wolf, F. G. Omenetto, X. Zhang, K. A. Nelson, and R. D. Averitt, Nature 487, 345 (2012).

[9] E. Pomarico, M. Mitrano, H. Bromberger, M. A. Sentef, A. Al-Temimy, C. Coletti, A. Stöhr, S. Link, U. Starke, C. Cacho, R. Chapman, E. Springate, A. Cavalleri, and I. Gierz, Phys. Rev. B 95, 024304 (2017).

[10] D. M. Kennes, E. Y. Wilner, D. R. Reichman, and A. J. Millis, Nat. Phys. 13, 479 (2017).

[11] M. A. Sentef, Phys. Rev. B 95, 205111 (2017).

[12] T. Oka and H. Aoki, Phys. Rev. B 79, 081406(R) (2009).

[13] T. Kitagawa, T. Oka, A. Brataas, L. Fu, and E. Demler, Phys. Rev. B 84, 235108 (2011).

[14] N. H. Lindner, G. Refael, and V. Galitski, Nat. Phys. 7, 490 (2011).

[15] M. A. Sentef, M. Claassen, A. F. Kemper, B. Moritz, T. Oka, J. K. Freericks, and T. P. Devereaux, Nat. Commun. 6, 7047 (2015).

[16] H. Hübener, M. A. Sentef, U. De Giovannini, A. F. Kemper, and A. Rubio, Nat. Commun. 8, 13940 (2017).

[17] Y. H. Wang, H. Steinberg, P. Jarillo-Herrero, and N. Gedik, Science 342, 453 (2013).

[18] F. Mahmood, C.-K. Chan, Z. Alpichshev, D. Gardner, Y. Lee, P. A. Lee, and N. Gedik, Nat. Phys. 12, 306 (2016).

[19] J. Struck, C. Ölschläger, R. Le Targat, P. Soltan-Panahi, A. Eckardt, M. Lewenstein, P. Windpassinger, and K. Sengstock, Science 333, 996 (2011).

[20] M. Aidelsburger, M. Atala, S. Nascimbène, S. Trotzky, Y.-A. Chen, and I. Bloch, Phys. Rev. Lett. 107, 255301 (2011).

[21] H. Miyake, G. A. Siviloglou, C. J. Kennedy, W. C. Burton, and W. Ketterle, Phys. Rev. Lett. 111, 185302 (2013).

[22] G. Jotzu, M. Messer, R. Desbuquois, M. Lebrat, T. Uehlinger, D. Greif, and T. Esslinger, Nature 515, 237 (2014).

[23] N. Fläschner, B. S. Rem, M. Tarnowski, D. Vogel, D.-S. Lühmann, K. Sengstock, and C. Weitenberg, Science 352, 1091 (2016).

[24] J. Näger, K. Wintersperger, M. Bukov, S. Lellouch, E. Demler, U. Schneider, I. Bloch, N. Goldman, and M. Aidelsburger, arXiv:1808.07462.

[25] K. W. Madison, M. C. Fischer, R. B. Diener, Q. Niu, and M. G. Raizen, Phys. Rev. Lett. 81, 5093 (1998).

[26] S. Longhi, M. Marangoni, M. Lobino, R. Ramponi, P. Laporta, E. Cianci, and V. Foglietti, Phys. Rev. Lett. 96, 243901 (2006).

[27] H. Lignier, C. Sias, D. Ciampini, Y. Singh, A. Zenesini, O. Morsch, and E. Arimondo, Phys. Rev. Lett. 99, 220403 (2007).

[28] M. C. Rechtsman, J. M. Zeuner, Y. Plotnik, Y. Lumer, D. Podolsky, F. Dreisow, S. Nolte, M. Segev, and A. Szameit, Nature 496, 196 (2013).

[29] M. Aidelsburger, S. Nascimbene, and N. Goldman, C. R. Phys. 19, 394 (2018).

[30] G. Jotzu, M. Messer, F. Görg, D. Greif, R. Desbuquois, and T. Esslinger, Phys. Rev. Lett. 115, 073002 (2015).

[31] C. V. Parker, L.-C. Ha, and C. Chin, Nat. Phys. 9, 769 (2013).

[32] C. J. Kennedy, W. C. Burton, W. C. Chung, and W. Ketterle, Nat. Phys. 11, 859 (2015).

[33] M. Aidelsburger, M. Lohse, C. Schweizer, M. Atala, J. T. Barreiro, S. Nascimbène, N. R. Cooper, I. Bloch, and N. Goldman, Nat. Phys. 11, 162 (2014).

[34] T. Ozawa, H. M. Price, A. Amo, N. Goldman, M. Hafezi, L. Lu, M. Rechtsman, D. Schuster, J. Simon, O. Zilberberg, and I. Carusotto, Rev. Mod. Phys. 91, 015006 (2019).
[35] L. Asteria, D. T. Tran, T. Ozawa, M. Tarnowski, B. S. Rem, N. Fläschner, K. Sengstock, N. Goldman, and C. Weitenberg, Nature Physics 15, 449 (2019).

[36] J. W. McIver, B. Schulte, F.-U. Stein, T. Matsuyama, G. Jotzu, G. Meier, and A. Cavalleri, arXiv:1811.03522.

[37] M. Bukov, L. D’Alessio, and A. Polkovnikov, Adv. Phys. 64, 139 (2015).

[38] L. E. F. Foa Torres, P. M. Perez-Piskunow, C. A. Balseiro, and G. Usaj, Phys. Rev. Lett. 113, 266801 (2014).

[39] G. Usaj, P. M. Perez-Piskunow, L. E. F. Foa Torres, and C. A. Balseiro, Phys. Rev. B 90, 115423 (2014).

[40] T. Mikami, S. Kitamura, K. Yasuda, N. Tsuji, T. Oka, and H. Aoki, Phys. Rev. B 93, 144307 (2016).

[41] H. Dehghani, T. Oka, and A. Mitra, Phys. Rev. B 90, 195429 (2014).

[42] H. Dehghani, T. Oka, and A. Mitra, Phys. Rev. B 91, 155422 (2015).

[43] A. Kundu, H. A. Fertig, and B. Seradjeh, Phys. Rev. Lett. 113, 236803 (2014).

[44] T. Meier, G. von Plessen, P. Thomas, and S. W. Koch, Phys. Rev. Lett. 73, 902 (1994).

[45] K. I. Seetharam, C.-E. Bardyn, N. H. Lindner, M. S. Rudner, and G. Refael, Phys. Rev. X 5, 041050 (2015).

[46] R. Hertel, J. Magn. Magn. Mater. 303, L1 (2006).

[47] P.-O. Löwdin, Phys. Rev. 97, 1474 (1955).

[48] M. Claassen, C. Jia, B. Moritz, and T. P. Devereaux, Nat. Commun. 7, 13074 (2016).

[49] D. Shin, H. Hübener, U. De Giovannini, H. Jin, A. Rubio, and N. Park, Nat. Commun. 9, 638 (2018).

[50] N. Tancogne-Dejean, M. A. Sentef, and A. Rubio, Phys. Rev. Lett. 121, 097402 (2018).

[51] J. Gong, L. Morales-Molina, and P. Hänggi, Phys. Rev. Lett. 103, 133002 (2009).

[52] R. Singla, G. Cotugno, S. Kaiser, M. Först, M. Mitrano, H. Y. Liu, A. Cartella, C. Manzoni, H. Okamoto, T. Hasegawa, S. R. Clark, D. Jaksch, and A. Cavalleri, Phys. Rev. Lett. 115, 187401 (2015).

[53] G. E. Topp, N. Tancogne-Dejean, A. F. Kemper, A. Rubio, and M. A. Sentef, Nat. Commun. 9, 4452 (2018).

[54] M. Claassen, D. M. Kennes, M. Zingl, M. A. Sentef, and A. Rubio, Nat. Phys. (2019), doi: 10.1038/s41567-019-0532-6.

[55] T. L. Cocker, D. Peller, P. Yu, J. Repp, and R. Huber, Nature 539, 263 (2016).

[56] M. Liu, A. J. Sternbach, M. Wagner, T. V. Slusar, T. Kong, S. L. Bud'ko, S. Kittiwatanakul, M. M. Qazilbash, A. McLeod, Z. Fei, E. Abreu, J. Zhang, M. Goldflam, S. Dai, G.-X. Ni, J. Lu, H. A. Bechtel, M. C. Martin, M. B. Raschke, R. D. Averitt, S. A. Wolf, H.-T. Kim, P. C. Canfield, and D. N. Basov, Phys. Rev. B 91, 245155 (2015).

[57] M. Breusing, S. Kuehn, T. Winzer, E. Malić, F. Milde, N. Severin, J. P. Rabe, C. Ropers, A. Knorr, and T. Elsaesser, Phys. Rev. B 83, 153410 (2011).

[58] D. Brida, A. Tomadin, C. Manzoni, Y. J. Kim, A. Lombardo, S. Milana, R. R. Nair, K. S. Novoselov, A. C. Ferrari, G. Cerullo, and M. Polini, Nat. Commun. 4, 1987 (2013).

[59] I. Gierz, F. Calegari, S. Aeschlimann, M. Chávez Cervantes, C. Cacho, R. T. Chapman, E. Springate, S. Link, U. Starke, C. R. Ast, and A. Cavalleri, Phys. Rev. Lett. 115, 086803 (2015).

[60] W. V. Houston, Phys. Rev. 57, 184 (1940).

[61] J. B. Krieger and G. J. Iafrate, Phys. Rev. B 33, 5494 (1986). 